\title{
NEUROPSICOLOGIA E DIAGNÓSTICO DIFERENCIAL NOS DECLÍNIOS COGNITIVOS E PROCESSOS DEMENCIAIS NO IDOSO
}

\section{ARTIGO DE REVISÃO}

VANZELER, Maria Luzinete Alves ${ }^{1}$

VANZELER, Maria Luzinete Alves. Neuropsicologia e diagnóstico diferencial nos declínios cognitivos e processos demenciais no idoso. Revista Científica Multidisciplinar Núcleo do Conhecimento. Ano 05, Ed. 09, Vol. 02, pp. 30-54. Setembro de 2020.

ISSN:

2448-0959,

Link de acesso: https://www.nucleodoconhecimento.com.br/psicologia/declinios-cognitivos

\section{RESUMO}

Com o objetivo de realizar a revisão da literatura sobre o declínio cognitivo no envelhecimento, normal e patológico e o papel da avaliação neuropsicológica no diagnóstico diferencial destes declínios, foi realizada uma busca em livros textos especializados, manuais de avaliação neuropsicológicas e artigos científicos indexados (nos Periódicos CAPES, Lilacs, PubMed, Mediline e Scielo e Google acadêmico), a revisão foi realizada em novembro e dezembro de 2019. O estudo reuniu informações a respeito dos déficits cognitivos no envelhecimento normal, no comprometimento cognitivo leve $(\mathrm{CCL})$ e em diferentes tipos de demências, como: Doença de Alzheimer (DA); Demência frontotemporal (DFT); Demência com corpúsculos de Lewy (DCL); Demência vascular (DV); Demência mista (DA/DV); demências associadas a outros distúrbios neurodegenerativos; demências infecciosas e demências reversíveis. Verificou-se a importância da avaliação neuropsicológica como ferramenta de diagnóstico, utilizando-se de recursos como: entrevistas, observações comportamentais, baterias de rastreamentos e testes específicos (uso exclusivo do psicólogo). A avaliação neuropsicológica caracteriza as demências como um progressivo déficit na função cognitiva, com prejuízo da memória

\footnotetext{
${ }^{1}$ Doutora em Patologia experimenta e comparada - USP-SP.
} 
desde a fase inicial, o que prejudica as atividades sociais e ocupacionais. Alem disso a avaliação neuropsicológica pode identificar situações em que há possibilidade de reversão, quadros primários e sequelares e ainda orientar terapia e indicar prognóstico.

Palavras-chave: avaliação neuropsicológica, funções cognitivas, demências, envelhecimento, idosos.

\section{INTRODUÇÃO}

O aumento na expectativa de vida é sem dúvida uma conquista da sociedade contemporânea. Segundo Burlá (2013) o grupo populacional que mais tem crescido em quase todo mundo é o das pessoas com idade igual ou superior a 60 anos. Esse crescimento está relacionado com a redução nas taxas de mortalidade por doenças infectocontagiosas e crônicas em todas faixas etárias. Além disso, o avanço da tecnologia médica, o maior acesso a serviços de saúde e a melhoria das condições de vida em geral, tem papel fundamental para o crescimento populacional nesta faixa etária (BURLÁ, 2013; KALACHE et al., 1987).

O Instituto Brasileiro de Geografia e Estatística (IBGE) (2010), registou o aumento do envelhecimento populacional no Brasil nas últimas décadas. Neste mesmo período foi registrado também, a redução da fecundidade, da natalidade e da mortalidade, associados as melhorias do saneamento básico, da alimentação e do desenvolvimento de fármacos (ZIMERMAN, 2000). Segundo o IBGE (2010), em 2050 - Brasil terá a quinta maior população idosa do mundo. Esse incremento da expectativa de vida, traz consigo um aumento a propensão às doenças crônicodegenerativas, tais como diabetes mellitus, hipertensão arterial, entre outras (CARAMELLI e BARBOSA, 2002). Além disso com o envelhecimento, surge o declínio cognitivo, que pode ocorrer de forma natural e fisiológica, ou pode significar um quadro patológico. Esse quadro pode ser caracterizado como demência, ou apresentar-se como comprometimento cognitivo leve (CCL) cuja magnitude não chega a interferir nas atividades de vida diária (AVD), porém indivíduos com CCL tem maior probabilidade de desenvolver demência. Desta forma o CCL pode ser 
considerado como um estágio intermediário entre o envelhecimento normal e um envelhecimento patológico (MALLMANN e DORING, 2017).

$\mathrm{Na}$ tentativa de entender cada vez mais o processo de envelhecimento populacional, a sociedade já inseriu a temática na formação de politicas públicas. A psicologia procura lidar com essa demanda, utilizando a atenção em saúde do idoso. Neste contexto a avaliação neuropsicológica tem papel fundamental, pois fornece subsídios para o diagnóstico precoce e promove medidas de intervenção que podem postergar a degeneração cognitiva (FRAGA, 2018).

Realizar avaliação neuropsicológica de pessoas idosas, mesmo que sem queixas cognitivas é um recurso muito importante, uma vez que algumas doenças neurodegenerativas existem bem antes de surgirem os sintomas de declínios funcionais ou cognitivos (BONDI et al., 2008; CARAMELLI e BEATO, 2008).

Rossini et al. (2007) e Yassuda et al. (2010) ressaltam a importância da investigação o mais cedo possível, para que o idoso com algum comprometimento possa se beneficiar das intervenções psicofarmacológicas e psicoterapêuticas. Damasceno (2006) enfatizou a avaliação neuropsicológica como um procedimento fundamental para conhecimento do perfil cognitivo de pessoas em geral, sobretudo, para diferenciar os diversos tipos demências.

Apesar dos benefícios a respeito da realização da avaliação neuropsicológica, a mesma ainda é uma prática pouco utilizada, fundamentalmente em se tratando de idosos saudáveis ou sem queixas cognitivas (FIGUEIREDO et al., 2009). Isto sugere a necessidade urgente de mais estudos sobre o envelhecimento e o papel da avaliação neuropsicológica neste contexto. Neste sentido o objetivo deste artigo é realizar um estudo descritivo do declínio cognitivo no envelhecimento, enfatizando as diferenças entre o processo normal, o comprometimento cognitivo leve e as demenciais, bem como descrever o papel da avaliação neuropsicológica no diagnóstico diferencial destes estados de vida. 


\section{MÉTODO}

Foi realizado um levantamento bibliográfico em artigos indexados no PUBMED, LILACS, MEDLINE, SCIELO e Google Acadêmico, bem como livros textos especializados e manuais de avaliação neuropsicológicas.

A revisão bibliográfica foi realizada nos meses novembro e dezembro de 2019, quando foram selecionados artigos, criticamente analisados. Para a busca dos artigos foram utilizados os seguintes descritores: Avaliação neuropsicológica, demências, funções cognitivas, idosos, envelhecimento.

\section{DESENVOLVIMENTO}

\subsection{NEUROPSICOLOGIA E AVALIAÇÃO NEUROPSICOLÓGICA}

A neuropsicologia é a área de fronteira de diversas disciplinas, possuindo assim caráter interdisciplinar, a medida que incorpora conceitos e técnicas de disciplinas básicas, como: neuroanatomia, neurofisiologia, neuroquímica e neurofarmacologia, e disciplinas de aplicação, como: psicometria, psicologia clínica e experimental, psicopatologia e psicologia cognitiva (RAMOS e HAMDAN, 2016).

O domínio neuropsicológico pode ser compreendido através de três vertentes complementares:

a) na primeira vertente, a neuropsicologia é entendida como uma disciplina clínica com finalidade de identificar o perfil de déficits cognitivos de pacientes que sofreram lesões cerebrais ou estão em processo de envelhecimento;

b) na segunda vertente, trata-se de uma disciplina neurocientífica, que estabelece correlações anátomo-clínicas, possibilitando melhor entendimento das operações elementares, da dinâmica e da plasticidade das funções cognitivas;

c) e a terceira vertente é caracterizada como uma disciplina cognitiva, pois considera o desempenho em testes e tarefas obtidos por sujeitos com lesões cerebrais, e em 
fase de envelhecimento, formula testes de hipótese a partir de teorias cognitivas fundamentadas em estudos realizados com pessoas saudáveis (SIÉROFF, 2009).

Em qualquer das três vertentes a avaliação neuropsicológica é ferramenta indispensável, uma vez que se constituí em um método que inclui diversos procedimentos sistematizados, no sentido de investigar e mapear as funções mentais e cognitivas relacionadas ao funcionamento do sistema nervoso central (SNC) (MICHALICK-TRIGINELLI, 2018).

A avaliação neuropsicológica analisa a presença de alterações comportamentais, consequentes de disfunção neurológica ou dificuldade cognitiva causada por transtornos do desenvolvimento, lesões cerebrais ou decorrente do processo de envelhecimento (COSTA et al., 2004).

Para Cunha (2008) a avaliação neuropsicológica é um tipo bastante complexo de avaliação psicológica, pois exige do profissional uma sólida fundamentação em psicologia clínica e familiaridade com psicometria bem como especialização e treinamento em contextos onde seja fundamental o conhecimento do SNC e de suas patologias.

Mader-Joaquim (2018) afirma que a avaliação neuropsicológica consiste em um exame detalhado, que tem a função de avaliar o desempenho cognitivo, linguístico, perceptual e psicomotor, objetivando relacionar este desempenho com as condições funcionais e estruturais do cérebro. Para isto são utilizadas técnicas de entrevistas, exames qualitativos e quantitativos, sendo, portanto, uma extensão refinada da observação clínica.

Para avaliação neuropsicológica são utilizados instrumentos que avaliam vários domínios cognitivos. Segundo, Lopes e Argimon (2017) são inclusos na avaliação as habilidades intelectuais (QI), habilidades acadêmicas (aritmética, leitura, etc.), atenção, flexibilidade mental, inibição de resposta, resolução de problemas, raciocínio, compreensão da linguagem, fluência verbal, memória verbal e, habilidades viso 
espaciais, velocidade e integração viso motora e velocidade de processamento cognitivo.

Para avaliar os diferentes domínios cognitivos, a Avaliação Neuropsicológica inclui vários instrumentos comuns a diversos profissionais de saúde, tais como entrevistas, observações e tarefas neuropsicológicas. No entanto, quando a Avaliação Neuropsicológica é realizada por neuropsicólogo com formação em psicologia, acrescentase a possibilidade de utilização de testes psicológicos. Conforme 0 § $1 \stackrel{0}{ }$ do Art. 13 da Lei no 4.119/62, testes psicológicos são de uso exclusivo de psicólogos. A regulamentação sobre seu uso foi publicada na Resolução CFP № 002/2003. Essa resolução determina as normas sob as quais os testes são criados, utilizados e comercializados (MICHALICK-TRIGINELLI, 2018).

\subsection{AVALIAÇÃO NEUROPSICOLÓGICA DE PESSOAS IDOSAS}

A avaliação neuropsicológica em pessoas idosas deve ser realizado com cautela, em especial quando se vai investigar situações diagnósticas, pois exige perícia do profissional em compreender os limites entre o envelhecimento normal, a presença de $\mathrm{CCL}$ e o surgimento de quadros demenciais, levando em consideração as particularidades individuais (FRAGA, 2018). Neste sentido Gil e Busse (2009) afirmam que:

O envelhecimento populacional trouxe preocupações para os clínicos, dentre estas, entender o que está por trás dos lapsos de memória. Os idosos frequentemente reclamam esquecer o nome de pessoas conhecidas, comentam que o nome de objetos está na ponta da língua e não vêm à tona, não se recordam do nome de lugares que frequentaram; sendo estas, queixas comuns dentro de uma lista cada vez mais numerosa. Basta saber, se estas dificuldades representam declínio cognitivo associado ao envelhecimento, se as queixas fazem parte do diagnóstico de Comprometimento Cognitivo Leve (CCL) ou se realmente representam uma condição degenerativa do sistema nervoso central. Déficit progressivo de memória e de pelo menos uma outra função cortical superior (linguagem, função executiva, praxia e função visual) perfaz o diagnóstico de demência que é clínico, mas que requer confirmação do prejuízo cognitivo, por avaliação neuropsicológica detalhada, principalmente nas fases iniciais, segundo os critérios de NINCDS-ADRDA. 
NINCDS-ADRDA, são critérios para o diagnóstico da doença de Alzheimer, propostos em 1984 pelo National Institute of Neurological and Communicative Disorders and Stroke (NINCDS) e pela Alzheimer's Disease and Related Disorders Association e são muito usados internacionalmente (MCKHANN, 1984). Estes critérios são determinantes para que a presença de déficit cognitivo e/ou suspeita de síndromes demenciais sejam confirmadas por exames neuropsicológicos, antes determinar um diagnóstico clínico de possível Alzheimer. Os critérios estabelecem também que o diagnóstico definitivo só pode ser confirmado através de um exame histopatológico (análise microscópica do tecido cerebral) e ainda indicam os domínios cognitivos que podem ser afetados pela doença de Alzheimer (DA) (BLACKER, 1994).

\subsection{TIPOS DE DECLÍNIO COGNITIVO NO ENVELHECIMENTO}

\subsubsection{DECLÍNIO NO ENVELHECIMENTO NATURAL}

O envelhecimento é um fenômeno que atinge a todos os seres humanos. Uma vez que é um processo dinâmico, progressivo, irreversível e ligado a fatores de natureza biológicos, psíquicos e sociais (BRITO e LITVOC, 2004).

O envelhecimento normal geralmente vem associado a um declínio cognitivo, porém sem comprometer significativamente as AVD. Este declínio provavelmente está relacionado com a redução da velocidade de processamento de informações e de mudanças em algumas habilidades cognitivas específicas, principalmente a memória, a atenção, e as funções executivas (PETERSEN et al., 2001). Decarli (2003) descreve a prevalência de queixas de memória em idosos como muito frequente variando de $22 \%$ a $56 \%$, e entre as alterações cognitivas provavelmente é a queixa mais frequente nesta população.

O envelhecer acarreta alterações na velocidade de processamento das informações, propiciando o aumento do tempo para processar dados de: leitura, compreensão e memorização (AIMEIDA e BERGER, 2007). Também é comum ser observada a dificuldade em atividades que exigem flexibilidade e velocidade mental durante o 
processamento de informações no indivíduo em processo de envelhecimento (DE SOUZA et al., 2010).

Clemente e Ribeiro-Filho (2008) descrevem sucintamente os principais tipos de memória, e as partes que podem estar sendo afetadas durante o envelhecimento, são elas:

a) memória episódica (envolvida com lembranças de uma pequena história, ou com o que comeu na noite anterior, ou o que fez no último aniversário);

b) memória semântica (relacionada com saber a cor de um leão ou distinguir um garfo e uma colher);

c) memória de procedimentos, (relacionada a dirigir um carro ou jogar futebol);

d) Memória de trabalho (envolvida com a realização de cálculos matemáticos).

Segundo De Vitta (2000 apud FECHINE e TROMPIERI, 2015) durante o processo de envelhecimento, o SNC é o sistema que sofre maior comprometimento, pois há redução no número de neurônios, alterações na velocidade de condução nervosa e na intensidade dos reflexos, bem como restrição das respostas motoras, do poder de reações e da capacidade de coordenação.

Gallahue et al., (2013) relatam que, entre os 20 e 90 anos de idade, o córtex cerebral sofre perda de 10 a $20 \%$ de massa. Já em outras partes do cérebro pode ocorrer prejuízo de até $50 \%$. Assim, no processo normal à medida que o cérebro envelhece, a atividade bioquímica, realizada por neurotransmissores, neuropeptídeos e hormônios cerebrais, também declinam.

\subsubsection{COMPROMETIMENTO COGNITIVO LEVE (CCL)}

No conceito de CCL, estão envolvidas as funções de memória, atenção, linguagem, funções executivas e habilidades visuoconstrutivas. Neste caso, além da cognição, 
deve-se levar em consideração outros elementos, como o desempenho da pessoa idosa e a ausência de um quadro demencial (PETERSEN, 2009).

O CCL em idosos, refere-se a condição em que há perda cognitiva em comparação com o envelhecimento normal (na mesma faixa etária), mas não se enquadram em critérios que caracterizem demência. Porém este construto, tem sofrido alterações em sua definição ao longo do tempo, e atualmente é caracterizado como estágio de transição entre o envelhecimento cognitivo normal e uma síndrome demencial (ARETOULI et al., 2013; PETERSEN, 2014; VAN DER MUSSELE et al., 2014).

Segundo Petersen et al. (2014), o critério para o CCL leva em conta a preservação do desempenho cognitivo global, sem prejuízo funcional que inviabilize a realização das AVD e há ausência de demência. Porém, artigos mais recentes vem revendo estes critérios, com base em observações de pacientes com CCL que apresentam mínimos déficits funcionais, principalmente na realização de tarefas mais complexas (FORLENZA et al., 2013).

Nesse contexto, o CCL passa a ser visto como uma síndrome clínica com perfis diferenciados. Sendo agregadas questões biológicas e genéticas, preditores de progressão e comorbidades (PETERSEN et al, 2014).

Atualmente estão descritos dois subtipos principais de CCL, o amnéstico e o não amnéstico. O subtipo amnéstico, caracteriza-se pelo declínio da memória, em especial a episódica, podendo existir de forma isolada (CCL amnéstico único domínio), ou apresentar déficits cognitivos menores em outros domínios como; linguagem, funções executivas, ou habilidades visuo-espaciais (CCL amnéstico múltiplos domínios) (ZHENG et al, 2012). Já no segundo subtipo (não amnéstico), a memória está preservada, havendo um ou múltiplos domínios comprometidos (FICHMAN, 2013).

\subsubsection{NEUROPSICOLOGIA DAS DEMÊNCIAS}

Define-se demência como uma síndrome de declínio cognitivo-comportamental que se manifesta pelo comprometimento de pelo menos, duas funções mentais, como memória, linguagem e habilidade visuoespacial, de intensidade 
suficientemente grave para comprometer a autonomia do paciente na realização das atividades de vida cotidiana (DE SOUSA e TEIXEIRA, 2014).

As principais causas de demência são as doenças neurodegenerativas. Sendo a doença de Alzheimer (DA) a causa mais frequente chegando a $60 \%$ de todas as demências (LOGIUDICE, 2002). Entre as causas neurodegenerativas menos frequentes encontram-se as demências de corpos de Levy (DCL) e a frontotemporal (DFT). Além das doenças neurodegenerativas existem as demências não degenerativas, a qual pertence a demência vascular (DV), responsável por cerca de $25 \%$ dos casos e outras menos comuns, conhecidas como demências secundárias que incluem processos infecciosas (neurossífilis associada ao HIV), processos metabólicos (hipotireoidismo e deficiência de vitamina B12), e estruturais (tumor, hematoma e hidrocefalia de pressão normal) (DE SOUSA e TEIXEIRA, 2014). Enfim, são diversas as condições médicas que estão associadas com a presença de sintomas demenciais, algumas delas serão descritas a seguir.

Doença de Alzheimer (DA) - A DA é uma doença de etiologia desconhecida, exceto em raros casos familiares, cujo início precoce, relaciona-se a mutação genética específica (HEYMAN et al., 1984). Caracteriza-se por uma progressiva dificuldade cognitiva, comportamental e funcional, principalmente na velhice (REITZ e MAYEUX, 2014) e corresponde a cerca de $60 \%$ dos casos de demências (GALLUCCI NETO et al., 2005). A forma típica da DA costuma surgir a partir dos 65 anos, com sintoma inicial caracterizado por por déficit progressivo de memória episódica anterógrada. Estes sintomas amnésicos estão diretamente relacionados com o acometimento de regiões temporais mediais, principalmente o hipocampo e o córtex entorrinal (DE SOUSA e TEIXEIRA, 2014). Já a evolução dos sintomas cognitivos e comportamentais da DA estão relacionadas ao avanço das alterações neurológicas, que em nível histopatológico, passa a apresentar emaranhados neurofibrilares e placas senis no cérebro que gradualmente levam à perda de sinapses e morte neuronal, cujas manifestações clínicas aparecem na forma de modificações cognitivas (VALLS-PEDRET et al., 2010). 
A DA tem evolução lenta e insidiosa, prejudicando de forma progressiva as funções neurocognitivas como; orientação, planejamento, memória, linguagem e juízo (HERRERA-RIVERO et al., 2010).

Segundo Cruz e Hamdan (2008), com a progressão da demência, o sujeito vai perdendo a capacidade de gerenciar as AVD (trabalho, estudo, lazer, vida social), e em estágios mais avançados, perde a capacidade de cuidar da higiene pessoal e alimentação, necessitando de ajuda de um cuidador. A gravidade dos sintomas aumenta conforme diferentes segmentos do cérebro vão sendo paulatinamente lesadas (HERRERA-RIVERO et al., 2010). Neste contexto a DA apresenta três estágios:

a) no primeiro estágio o dado clínico predominante é a perda de memória para fatos recentes, havendo preservação da memória para fatos remotos. O paciente apresenta dificuldade para armazenar e recuperar informações aprendidas a poucos minutos. Esquece nomes, não lembra onde guardou objetos pessoais e repete a mesma pergunta várias vezes. Porém as habilidades cognitivas tipo linguagens e praxias estão preservadas;

b) no estágio moderado o comprometimento alcança outros domínios cognitivos como (linguagem, memórias recente e remotas, habilidades visoespaciais, bem como as praxias visuais). Nessa etapa os pacientes perdem-se dentro da própria casa e tem dificuldade para se vestir e realizar higiene pessoal, sendo necessário auxilio para realização das AVD;

c) no estágio grave, todas as funções cognitivas estão gravemente prejudicadas, apresenta ecolalia, palilalia e mutismo, incontinência esfincteriana, pode apresentar rigidez generalizada, dificuldade para deglutir e restrição ao leito (DE SOUSA e TEIXEIRA, 2014).

Segundo Poulin e Zakzanis (2002), os estudos em vida, ainda são insipientes, faltam marcadores específicos para investigação laboratorial e de imagem. 
Outros autores verificaram a dificuldade em relação a um diagnóstico definitivo de DA, e que o mesmo só pode ser feito a partir da investigação histopatológica do Cérebro post-mortem (BRAAK e BRAAK, 1991).

Demência frototemporal (DFT) - Consiste de um agrupamento de síndromes neurodegenerativas, que comprometem sucessivamente o comportamento e/ou a linguagem. A DFT progride mais rapidamente que outras doenças neurodegenerativas e é caracterizada pela presença de alterações no movimento e/ou na linguagem, e mudanças progressivas do comportamento associadas a danos nas funções executivas. A palavra frontotemporal é uma consequência da degeneração do córtex frontal e temporal, em presença de conservação das áreas posteriores do cérebro no início da demência. A DFT se manifesta mais frequentemente, na faixa dos 50 a 60 anos de idade (FERNANDEZ-MATARRUBIA et al., 2014; GHOSH e LIPPA, 2015). Porém em um significativo número de pessoas a manifestação se dá após os 80 anos (DE SOUSA e TEIXEIRA, 2014).

Clinicamente a DFT, desde seu início é caracterizado por sintomas neuropsiquiátricos, vinculados a alteração da personalidade e transtorno de conduta social. Nesse quadro apresentam-se sintomas comportamentais como, impulsividade, desinibição, indiferença afetiva, apatia e perda de regras sociais.

Estes sintomas estão relacionados com as três áreas do lobo frontal (orbital, medial e dorsal), e sua interação com circuitos paralelos entre eles, os núcleos da base e o tálamo (TEIXEIRA e CARDOSO, 2004).

O comportamento orbital estaria associado a desinibição, impulsividade e comportamentos antissociais e estereotipados. Os comportamentos estereotipados ou ritualísticos podem assumir diferentes formas, desde estereotipias motoras e verbais simples, como repetir frequentemente gestos e palavras, a rotinas complexas. O comprometimento medial (córtex do cíngulo) correlaciona-se com apatia, passividade e tendência ao isolamento social. Conforme a progressão do processo degenerativo para a convexidade do lobo frontal e consequente disfunção dorsolateral, surgem alterações de funções executivas (DE SOUSA e TEIXEIRA, 2014).

Segundo Le Ber et al. (2006), clinicamente a DFT não é homogênea, podendo apresentar três padrões comportamentais diferenciados que são; as formas apática 
(inerte), a forma desinibida, e a mixta. As formas apática e desinibida são em particular distinguíveis em estágios iniciais, já em estágios avançados é comum a sobreposição das formas.

A avaliação neuropsicológica é um instrumento útil ao diagnóstico de DFT, pois investiga peculiaridades comportamentais e cognitivas que possibilitam a obtenção de diagnósticos diferenciais (VALVERDE et al., 2009). Já o reconhecimento de atrofia frontal ou temporal, através de neuroimagem, é insuficiente para definir um quadro de DFT (NEARY et al., 2005).

Demência com corpúsculos de Lewy (DCL) - é uma demência neurodegenerativa, que apresenta declínio cognitivo flutuante, combinado com sintomas extrapiramidais e alucinações visuais. As flutuações do estado mental apresentam episódios recorrentes de delirium (estado confusional agudo), com interferência no nível da consciência e atenção, sem causa aparente e com duração variável (DE SOUSA e TEIXEIRA, 2014; GALLUCCI NETO, 2005).

As características demenciais da DCL tem inicio rápido e apresentam déficits perceptíveis nas funções executivas, resolução de problemas, fluência verbal e performance audiovisual. Apresentam sintomas de parkinsonismo como, hipomimia, bradicinesia, rigidez e tremor de repouso. Porém os neurolépticos, podem agravar o quadro de parkinsonismo em vez de melhorar. Nessa classificação estão excluídos pacientes com doença de Parkinson que se tornaram demenciados (GALVIN, 2003).

O perfil neuropsicológico dos pacientes com DCL, associa déficits corticais e subcorticais. Segundo Cahn-Weiner et al. (2003) a presença de déficits atencionais, visoespaciais e visoconstrutivos são bastante acentuados na DCL. Esses autores, realizaram o teste do desenho do relógio com finalidade de discriminar DCL em relação a DA, e verificaram que embora os escores para o desenho espontâneo tenham sido similares, na cópia do desenho o desempenho dos pacientes com DA tendiam a melhorar e os pacientes com DCL tendem a manter os erros de organização espacial. Porém a memória episódica é frequentemente menos comprometida nos 
quadros de DCL, e em testes atencionais os pacientes DCL beneficiam-se de pistas (DE SOUSA e TEIXEIRA, 2014).

A fisiopatologia específica dessa demência, é a presença de corpúsculos de Lewy, que são inclusões eosinofílicas hialinas, presentes no córtex cerebral e tronco encefálico de modo geral, e eventos patológicos relacionados a DA, como placas senis e em menor proporção emaranhados de neurofibrilas (GALVIN, 2003).

Demência vascular (DV) - As DV são responsáveis por cerca de $25 \%$ dos casos de demências, sendo considerada a segunda maior causa de demência (KALARIA et al., 2008). Seu diagnóstico depende da relação causal entre síndrome demencial e a doença cerebrovascular. Assinala-se a heterogeneidade clínica na DV, já que os quadros da doença cerebrovascular variam muito, compreendendo desde doença de pequenos vasos (lesões lacunares e lesões de substância branca), a doença de grandes vasos (lesões corticais focais, ou múltiplas lesões de territórios vasculares limítrofes) até lesões hemorrágicas intracerebrais (BOWLER, 2003; AURIACAMB et al., 2008).

Ao definir demência classicamente prioriza-se o comprometimento de memória (por acometimento cortical, como na DA (APA, 2000). No entanto grande número de pacientes, apresentam comprometimento primário em outros domínios cognitivos. Os Pacientes com DV são acometidos por síndrome demencial córtico-subcortical, apresentando sintomas iniciais de déficits nas funções executivas ou focais múltiplas (ROMÁN, 2002).

Demência mista - esta entidade nosológica é conhecida por apresentar eventos típicos de DA e DV ao mesmo tempo. Estudos patológicos, tem sugerido que mais de um terço dos pacientes com DA apresentem concomitantemente lesões vasculares, $e$ a mesma proporção de pacientes com DV apresentam alterações patológicas características de DA (KALARIA e BALLARD, 1999). 
Há um tipo de demência mista em que o paciente com sintomas clínicos de DA, apresenta piora abrupta associada a sinais clínicos de acidente vascular cerebral (AVC) esta é descrita como demência pré-AVC (HÉNON et al., 2001)

Demências associadas a outros distúrbios neurovegetativos - a) Doença de Huntington (DH), quadro em que a memória é afetada em todos os aspectos, e mais tardiamente surge afasia, apraxia, agnosia e disfunção cognitiva global; b) Doença de Parkinson; c) Doença de Creutzfeldt-Jakob (DCJ), é o protótipo de doença causada por príons, sendo uma enfermidade infecciosa e sempre fatal (GALLUCCI NETO et al., 2005).

Demências infecciosas e outros agentes causadores - Alcoolismo; Neurocisticercose; Encefalite herpética; Complexo aids-demência; Neurossífilis; Síndrome de Wernicke-Korsakoff (GALLUCCI NETO et al., 2005).

Demências reversíveis - as demências reversíveis ocorrem mais raramente. Porém, seu diagnóstico é muito importante, uma vez que o tratamento adequado pode reverter o declínio cognitivo. As mais conhecidas são: a) Hidrocefalia de pressão normal (HPN); b) Pelagra (causada pela deficiência de ácido nicotínico (niacina)); c) Deficiência de vitamina B12; d) Hipotireoidismo e; e) Depressão (GALLUCCI NETO et al., 2005).

\subsection{PROCESSO DE AVALIAÇÃO NEUROPSICOLÓGICA NO IDOSO}

Em função do declínio cognitivo e da maior suscetibilidade à distúrbios neuropsiquiátricos nos idosos, se faz necessário um estudo integral, envolvendo avaliações de parâmetros como: linguagem, coordenação motora, condições de percepção e sensorial, capacidade de abstração, raciocínio, atenção, cálculo e memória.

O exame neuropsicológico é imprescindível na clínica geriátrica. Conforme descrição de Morais et al. (2010): 
O exame neuropsicológico apresenta inúmeras aplicações em geriatria e tem por objetivo a aplicação de testes psicométricamente validados e padronizados para avaliação cognitiva, funcional e comportamental, identificando síndromes, demenciais, transtornos psiquiátricos e déficits cognitivos, bem como o impacto nas atividades, laborais, sociais, familiares e de lazer.

A avaliação cognitiva é fundamental tanto para o diagnóstico quanto para o planejamento e execução de medidas terapêuticas conforme descrito no texto abaixo.

A realização da avaliação cognitiva pode ser um instrumento útil na avaliação global do paciente idoso, permitindo ao clínico geral, psiquiatra, neurologista ou geriatra obterem informações que subsidiem tanto o diagnóstico etiológico do quadro em questão, quanto o planejamento e execução das medidas terapêuticas e de reabilitação a serem realizadas em cada caso. Infelizmente, essa não é uma prática de rotina no Brasil em serviços de saúde em atenção primária, e mesmo secundária, seja em psiquiatria, em neurologia ou geriatria (AZAMBUJA, 2007).

A avaliação de rastreamento cognitivo é de fundamental importância na avaliação da saúde mental da população idosa, já que a mesma apresenta maior vulnerabilidade para desenvolvimento de demência, havendo uma crescente preocupação com a detecção de problemas que comprometem sua autonomia e capacidade funcional (AZAMBUJA, 2007; SCHLINDWEIN-ZANINI, 2010).

Uma avaliação neuropsicológica de idosos envolve diversos passos, incluindo (anamnese, observação comportamental, teste gerais de rastreamento neuropsicológico, investigação da funcionalidade do indivíduo e avaliação específica). Nos próximos itens serão descritos a sequência de avaliação neuropsicológica de forma geral.

\subsubsection{ANAMNESE}

A avaliação do paciente tem início com a anamnese associada às observações comportamentais. A anamnese está orientada para obtenção de dados históricos e sistematização dos motivos da consulta e sintomas atuais (CARRETONI FILHO e PREBIANCHI, 2011). 


\subsubsection{BATERIAS DE RASTREAMENTO NEUROPSICOLÓGICAS}

Após a anamnese recomenda-se a realização de baterias neuropsicológicas de rastreamento, uma vez que estas são sequências de testes simples e rápidos com finalidade de avaliar a condição comportamental e cognitiva, do indivíduo. As baterias são de fundamental importância para o estabelecer um perfil cognitivo basal, o perfil de evolução durante e depois dos tratamentos, bem como, auxiliam o diagnóstico diferencial quando há prejuízo cognitivo (SPREEN, 1998). Entre esses instrumentos a bateria CERAD (Consortium Estabilish of Alzheimer Disease) (MORRIS et al., 1993), que é bastante utilizada, pois avalia a memória (fixação, evocação e reconhecimento), linguagem (nomeação e fluência verbal), praxia (cópia de desenhos geométricos) e funções executivas (teste das trilhas).

O Mini Exame do Estado Mental (MEEM), é um instrumento de avaliação neuropsicológica importante especialmente para avaliação preliminar dos distúrbios cognitivos de pessoas idosas com mais de 8 anos de escolaridade (BERTOLUCCI et al., 2001).

A escala de Mattis para avaliação de demência (MDRS) (MATTIS, 1988) que é composta por 36 itens, distribuídos em cinco subescalas que são: atenção, iniciação e perseveração, construção, conceituação e memória. A soma das cinco subescalas representa o grau de comprometimento cognitivo e/ou da gravidade do quadro demencial (PORTO et al., 2003).

O teste do desenho do relógio é um recurso rápido (dura em média 3 a 5 minutos) e reproduz o tipo de funcionamento frontal e temporoparietal. Pacientes que apresentam escores normais no MEEM podem apresentar severas limitações funcionais no teste do relógio, indicando que as disfunções executivas podem anteceder os distúrbios de memória nas demências (ATALAIA-SILVA e LOURENCO, 2008).

O teste de fluência verbal (FV) é uma tarefa cognitiva complexa e muito sensível a todo tipo de dano cerebral, apontando, precocemente, os processos de deterioração 
executiva. O teste de FV mais utilizado é o de categoria semântica (animais/minuto), este é recomendado pelo Departamento Científico de Neurologia Cognitiva e do Envelhecimento, da Academia Brasileira de Neurologia (NITRINI et al., 2005).

\subsubsection{INSTRUMENTOS DE INVESTIGAÇÃO DE FUNCIONALIDADE}

Nesta categoria estão diversos instrumentos utilizados para avaliar a funcionalidade do idoso em relação a independência funcional, e as AVD. Os instrumentos visam medir o desempenho em atividades básicas (alimentação, banho, vestuário, higiene pessoal, eliminações intestinais, eliminações vesicais, uso do vaso sanitário, passagem cadeira-cama, deambulação e escadas) entre os testes estão: a) índice de Barthel que é um instrumento amplamente usado no mundo para a avaliação da independência funcional e avalia AVD (MINOSSO et al., 2010); b) índice de Katz, também chamado Índice de Atividades Básicas de Vida Diária (ABDV) foi desenvolvido por Sidney Katz, e até hoje é um dos instrumentos mais utilizados nos estudos de gerontologia nacional e internacional (DUARTE et al., 2007); c) índice de autocuidado de Kenny, é um instrumento de avaliação das atividades instrumentais de vida diária, suas tarefas estão agrupadas em seis categorias: locomoção, transferências, atividades básicas, vestuário, higiene pessoal e alimentação (RIBEIRO, 2017); d) avaliação geriátrica ampla (AGA); é um processo diagnóstico multidimensional, sistemático, cujo objetivo é determinar a fragilidade do idoso em relação a problemas médicos, psicossociais e funcionais, e planejar um tratamento e acompanhamento global e de longo prazo (RIBEIRO, 2017); e) avaliação funcional breve do idoso (AFB): é um instrumento de investigação de indícios de perdas da funcionalidade que impliquem em prejuízos na realização de atividades diárias (RIBEIRO, 2017).

\subsubsection{INSTRUMENTOS NEUROPSICOLÓGICOS ESPECÍFICOS}

Realizada a anamnese, e observações comportamentais iniciais segue-se a testagem através de baterias gerais de rastreamentos e investigação sobre a funcionalidade do idoso e ao final obtém-se o perfil geral do indivíduo. A partir deste perfil, pode-se ampliar a investigação utilizando-se os testes que sejam necessários para 
esclarecimentos e diagnósticos diferenciais. Aqui serão descritos alguns dos testes mais comumente utilizados:

a) Escala Weschsler abreviada de inteligência (WASI), por fornecer os tradicionais escores de QI Verbal, QI de Execução e QI Total e avaliar aspectos cognitivos, como: conhecimento verbal, processamento de informação visual, raciocínio espacial e não verbal, inteligência fluída e cristalizada em diversos contextos (TRENTINI et al., 2014);

b) Bateria psicológica para avaliação de atenção (BPA), que tem o objetivo avaliar a capacidade geral de atenção, bem como proceder avaliação individualizada dos tipos de atenção específicos (Atenção Concentrada, Dividida e Alternada) (RUEDA, 2013);

c) Teste de aprendizagem auditivo-verbal de Rey (RAVLT) este teste tem a finalidade avaliar a memória declarativa episódica utilizando a repetição de uma lista de palavras. É um teste é mais apropriado para pacientes com transtornos mentais e doenças neurológicas em especial transtornos neurocognitivos (demência e CCL). (DE PAULA e MALLOY-DINIZ, 2018);

d) Figuras complexas de Rey - este instrumento pode ser utilizado com finalidade de investigar memória visual, habilidade viso-espacial e certas funções de planejamento e execução de ações (DIAS, 2014).

Além destes muitos outros testes podem ser escolhidos para avaliação, dependendo do que foi detectado na avaliação inicial.

\section{CONCLUSÃO}

Com a população cada vez mais envelhecida, a avaliação neuropsicológica dos adultos e idosos, assume papel fundamental, pois quanto mais precoce for a identificação de alterações no sistema cognitivo (memória, linguagem, percepção e funções executivas) e suas relações (desde comportamentos simples até os de maior complexidade que exigem muito do cérebro), mas cedo serão feitas intervenções terapêuticas. Sendo possível interferir na qualidade de vida dos portadores de déficits, 
retardar a evolução clínica e até mesmo reverter os quadros demências consideradas reversíveis, reduzindo a dramaticidade dos danos durante a evolução clínica.

A avaliação neuropsicológica permite a identificação precoce dos distúrbios cognitivos, sua quantificação e diferenciação, o acompanhamento durante a evolução, e qualifica as intervenções terapêuticas.

Para maior segurança, a análise deve sempre levar em consideração a escolaridade do paciente, estados pré-mórbidos, uso de medicações ou outras substâncias que atuam no SNC. Além disso deve-se considerar os dados obtidos na anamnese, a impressão nas observações do comportamento, bem como os dados obtido nas baterias de rastreamentos e nos testes de avaliação referentes as limitações do paciente. Todas essas informações serão norteadoras para as escolhas dos testes adequados e darão mais segurança ao diagnóstico diferencial de CCL, tipos de demência, ou ainda indicar se o resultado obtido faz de um declínio natural.

Ao final, foram descritos o declínio cognitivo natural, o $C C L$, e os diversos tipos de demências, bem como a importância da avaliação neuropsicológica como ferramenta de diagnóstico diferencial nos déficits cognitivos, estando de acordo com os objetivos propostos. Dada a importância da avaliação neuropsicológica, sugere - se que novos estudos sejam realizados nesta área, no sentido de buscar novas técnicas, que aumentem a possibilidade de alcançar resultados mais precisos, e respostas mais objetivas, com consequências positivas para os pacientes.

\section{REFERÊNCIAS}

ALMEIDA, M., et. al., Oficina de memória para idosos: estratégia para promoção da saúde. Interface-Comunicação, Saúde, Educação, v. 11, p. 271-280, 2007.

AMERICAN PSYCHIATRIC ASSOCIATION (APA) - Dementia. In: DSM-IV. 4.ed. Washington. DC: American Psychiatric Association, p.147-71, 2000

ARETOULI, E., et. al., Four-year outcome of mild cognitive impairment: The contribution of executive dysfunction. Neuropsychology, v. 27, n.1, p. 95, 2013. 
ATALAIA-SILVA, K.; LOURENÇO, R. Tradução, adaptação e validação de construto do Teste do Relógio aplicado entre idosos no Brasil. Revista de Saúde Pública, v. 42, n. 5, p. 930-937, 2008.

AURIACOMBE, S. et al. Mise au point sur les demences vasculaires Update on vascular dementias. revue neurologique, v. 164, p. 22-41, 2008.

AZAMBUJA, Luciana Schermann. Avaliação neuropsicológica do idoso. Revista Brasileira de Ciências do Envelhecimento Humano, v. 4, n. 2, p. 40-45, 2007.

BERTOLUCCI, Paulo Henrique Ferreira et al. Applicability of the CERAD neuropsychological battery to Brazilian elderly. Arquivos de neuro-psiquiatria, $v$. 59, n. 3, p. 532-536, 2001.

BLACKER, Deborah, et al. Reliability and validity of NINCDS-ADRDA criteria for Alzheimer's disease: The National Institute of Mental Health Genetics Initiative. Archives of neurology, v. 51, n. 12, p. 1198-1204, 1994.

BONDI, Mark W., et al. Neuropsychological contributions to the early identification of Alzheimer's disease. Neuropsychology review, v. 18, n. 1, p.73-90, 2008.

BOWLER, J. V. Vascular cognitive impairment. Stroke, v. 35, n. 2, p. 386-388, 2004.

BRAAK, Heiko; BRAAK, Eva. Neuropathological stageing of Alzheimer-related changes. Acta neuropathologica, v. 82, n. 4, p. 239-259, 1991.

BRITO, F., et. al., Envelhecimento - prevenção e promoção de saúde. São Paulo: Atheneu, 2004. p.116.

BURLÁ, Claudia, et al. Panorama prospectivo das demências no Brasil: um enfoque demográfico. Ciência \& Saúde Coletiva, v. 18, p. 2949-2956, 2013. 
CAHN-WEINER, Deborah A., et al. Discrimination of dementia with Lewy bodies from Alzheimer disease and Parkinson disease using the clock drawing test. Cognitive and behavioral neurology, v. 16, n. 2, p. 85-92, 2003.

CARAMELLI, P.; BARBOSA, M. Como diagnosticar as quatro causas mais freqüentes de demência? How to diagnose the four most frequent causes of dementia? Revista Brasileira de Psiquiatria, v. 24, n. 1, p. 7-10, 2002.

CARAMELLI, P.; BEATO, R. Subjective memory complaints and cognitive performance in a sample of healthy elderly. Dementia \& Neuropsychologia, v. 2, n. 1, p. 42-45, 2008.

CARRETONI FILHO, H.; PREBIANCHI, M. Exame clinico psicológico (anamnese). $3^{\mathrm{a}}$ ed. São Paulo: Itatiba, 2011.

CLEMENTE, R.; RIBEIRO-FILHO, S. Comprometimento cognitivo leve: aspectos conceituais, abordagem clínica e diagnóstica. Revista Hospital Universitário Pedro Ernesto, v. 7, n. 1, p. 68-77, 2008.

COSTA, D. et al. Avaliação neuropsicológica da criança. Jornal de Pediatria, v. 80, n. 2, p. 111-116, 2004.

CRUZ, M.; HAMDAN, A. 0 impacto da doença de Alzheimer no cuidador. Psicologia em estudo, v. 13, n. 2, p. 223-229, 2008.

CUNHA, J. O ABC da avaliação neuropsicológica. In: CUNHA, Jurema Alcides, et al. (org.) Psicodiagnóstico. 5, ed. Porto Alegre: Artmed, 2008. p. 171-176.

DAMASCENO, B. Comprometimento Cognitivo Leve e Doença de Alzheimer Incipiente. In: CAIXETA, Leandro (ed.). Demência, abordagem Multidisciplinar. São Paulo, SP: Atheneu, 2006 p. 202-209.

DECARLI, C. Mild cognitive impairment: prevalence, prognosis, aetiology, and treatment. The Lancet Neurology, v. 2, n. 1, p. 15-21, 2003. 
DIAS, C. Avaliação neuropsicológica das funções executivas em um adulto com sintomas de ansiedade. Monografia (Especialização em Neuropsicologia ). Instituto de Psicologia, Universidade Federal do Rio Grande do Sul, Porto Alegre. 2014.

DUARTE, Y.; et. al. O Índex de Katz na avaliação da funcionalidade dos idosos. Revista da Escola de Enfermagem da USP, v. 41, n. 2, p. 317-325, 2007.

FERNÁNDEZ-MATARRUBIA, M., et al. Behavioural variant frontotemporal dementia: Clinical and therapeutic approaches. Neurología (English Edition), v. 29, n. 8, p. 464-472, 2014.

FICHMAN, H. Predomínio de Comprometimento Cognitivo Leve Disexecutivo em idosos atendidos no ambulatório da geriatria de um hospital público terciário na cidade do Rio de Janeiro. Neuropsicologia Latinoamericana, v. 5, n. 2, p. 31-40, 2013.

FIGUEIREDO, S., et al. Investigação neuropsicológica de uma amostra de sujeitos idosos saudáveis. Psicologia Hospitalar, v. 7, n. 2, p. 85-99, 2009.

FORLENZA, O., et al. Mild cognitive impairment (part 1): clinical characteristics and predictors of dementia. Brazilian Journal of Psychiatry, v. 35, n. 2, p. 178-185, 2013.

FRAGA, V. Avaliação Neuropsicológica em Idosos. Psicologia. pt-O Portal dos Psicólogos, 2018. Disponível em: $<$ https://www.psicologia.pt/artigos/ver_artigo_licenciatura.php?avaliacaoneuropsicologica-em-idosos\&codigo=TL0456\&area=>, Acesso em: 05 janeiro 2020.

GALVIN, J. Dementia with Lewy bodies. Archives of neurology, v. 60, n. 9, p. 13321335, 2003.

GALLAHUE, D.; et. al. Compreendendo o desenvolvimento motor: bebês, crianças, adolescentes e adultos. 7a Edição Porto Alegre RS: AMGH Editora, 2013. 
GALLUCCI N. José, et al. Diagnóstico diferencial das demências. Archives of Clinical Psychiatry (São Paulo), v. 32, n. 3, p. 119-130, 2005.

GHOSH, S.; LIPPA, C. Clinical subtypes of frontotemporal dementia. American Journal of Alzheimer's Disease \& Other Dementias, v.30, n. 7, p. 653-661, 2015.

GIL, G.; BUSSE, A. Avaliação neuropsicológica e o diagnóstico de demência, comprometimento cognitivo leve e queixa de memória relacionada à idade. Arquivos Médicos dos Hospitais e da Faculdade de Ciências Médicas da Santa Casa de São Paulo, v. 54, n. 2, p. 44-50, 2009.

HENON, H. et al. Poststroke dementia: incidence and relationship to prestroke cognitive decline. Neurology, v. 57, n. 7, p. 1216-1222, 2001.

HERRERA-RIVERO, M., et. al. Enfermedad de Alzheimer: inmunidad y diagnóstico. Revista de neurología, v. 51, n. 3, p. 153-164, 2010.

HEYMAN, A., et al. Alzheimer's disease: a study of epidemiological aspects. Annals of Neurology: Official Journal of the American Neurological Association and the Child Neurology Society, v. 15, n. 4, p. 335-341, 1984.

INSTITUTO BRASILEIRO DE GEOGRAFIA E ESTATÍSTICA (IBGE). Projeção da população do Brasil, Rio se janeiro, 2010. Disponível em: <ftp://ftp.ibge.gov.br/Censos/Censo_Demografico_2010/Caracteristicas_Gerais Religião Deficiência>, Acesso em: 05 janeiro 2020.

KALACHE, A., et al. The ageing of the world's population: a new challenge. Revista de saúde pública, v. 21, n. 3, p. 200-210, 1987.

KALARIA, R.; BALLARD, C. - Overlap Between Pathology of the Alzheimer Disease and Vascular Dementia. Alzheimer Dis Assoc Disord, v. 13, n. 3, p. 115123, 1999. 
KALARIA, R. et al. Alzheimer's disease and vascular dementia in developing countries: prevalence, management, and risk factors. The Lancet Neurology, v. 7, n. 9, p. 812-826, 2008.

LE BER, I., et al. Demographic, neurological and behavioural characteristics and brain perfusion SPECT in frontal variant of frontotemporal dementia. Brain, $v$. 129, n. 11. p. 3051-3065, 2006.

LOGIUDICE, D. Dementia: an update to refresh your memory. internal medicine journal. V.32, n.11, p.535-540, 2002.

LOPES, R.; ARGIMON, I. Avaliação Neuropsicológica Infantil: Aspectos Históricos, Teóricos e Técnicos. In: TISSER, Luciana et al. Avaliação neuropsicológica Infantil. Novo Hamburgo. Sinopsys, 2017.

MADER-JOAQUIM, M. O neuropsicólogo e seu paciente: a construção de uma prática. In: MALLOY-DINIZ, Leandro F., et al. (org.). Avaliação Neuropsicológica. 2. Ed. Porto Alegre: Artmed, 2018. p. 10-16

MALLMANN, M.; DORING, M. Aplicabilidade de uma bateria neuropsicológica em idosos com comprometimento cognitivo leve e doença de Alzheimer. Saúde e Pesquisa, v. 10, n. 3, p. 405-415, 2017.

MATTIS, S. Dementia rating scale: Professional manual. Florida: Psychological assessment resources, INC, 1988.

MCKHANN, Guy, et al. Clinical diagnosis of Alzheimer's disease: Report of the NINCDS-ADRDA Work Group* under the auspices of Department of Health and Human Services Task Force on Alzheimer's Disease. Neurology, v. 34, n. 7, p. 939939, 1984.

MICHALICK-TRIGINELLI, M., et al. Avaliação neuropsicológica: o papel da pesquisa na aprendizagem do psicólogo em formação. Pretextos-Revista da Graduação em Psicologia da PUC Minas, v. 3, n. 6, p. 309-327, 2018. 
MINOSSO, J., et al . Validação, no Brasil, do Índice de Barthel em idosos atendidos em ambulatórios. Acta paulista de Enfermagem, v. 23, n. 2, p. 218223, 2010.

MORAES, E., et al. Geriatria. In: MALLOY-DINIZ, L. $\mathrm{F}$ et al. Avaliação Neuropsicológica, Porto Alegre: Artmed, 2010 p.266.

MORRIS, J.; MOHS, R.; ROGERS, H. Consortium To Establish a Registry for Alzheimer's Disease (CERAD) Clinical and Neuropsychological. Psychopharmacology bulletin, v. 24, n. 3-4, p. 641, 1989.

NEARY, D.; SNOWDEN, J.; MANN, D. Frontotemporal dementia. The Lancet Neurology, v. 4, n. 11, p. 771-780, 2005.

NITRINI, R., et al. Diagnóstico de doença de Alzheimer no Brasil: avaliação cognitiva e funcional. Recomendações do Departamento Científico de Neurologia Cognitiva e do Envelhecimento da Academia Brasileira de Neurologia. Arquivos de Neuro-psiquiatria, v. 63, n. 3, p. 720-727, 2005.

PETERSEN, R. et al. Practice parameter: early detection of dementia: mild cognitive impairment (an evidence-based review): report of the Quality Standards Subcommittee of the American Academy of Neurology. Neurology, v. 56, n. 9, p. 1133-1142, 2001.

PETERSEN, R. et al. Mild cognitive impairment: ten years later. Archives of neurology, v. 66, n. 12, p. 1447-1455, 2009.

PETERSEN, R. et al. Mild cognitive impairment: a concept in evolution. Journal of internal medicine, v. 275, n. 3, p. 214-228, 2014.

PORTO, C. et al. Brazilian version of the Mattis dementia rating scale: diagnosis of mild dementia in Alzheimer's disease. Arquivos de neuro-psiquiatria, v. 61, n. 2, p. 339-345, 2003. 
POULIN, P.; ZAKZANIS, K. - In Vivo Neuroanatomy of Alzheimer's Disease: Evidence from Structural and Functional Brain Imaging. Brain Cognitive, v. 49, n. 2, p. 220-225, 2002.

RAMOS, A.; HAMDAN, A. O crescimento da avaliação neuropsicológica no Brasil: uma revisão sistemática. Psicologia: Ciência e Profissão, v.36, n. 2, p. 471- 485, 2016

REITZ, C.; MAYEUX, R. Alzheimer disease: epidemiology, diagnostic criteria, risk factors and biomarkers. Biochemical pharmacology, v. 88, n. 4, p. 640-651, 2014.

ROBERTS, R.; KNOPMAN, D. Classification and epidemiology of MCl. Clinics in geriatric medicine, v. 29, n. 4, p. 753-772, 2013

ROMÁN, G. Defining dementia: clinical criteria for the diagnosis of vascular dementia. Acta Neurologica Scandinavica. v.106, s.178, p.6-9, 2002.

RUEDA, F. Bateria Psicológica para Avaliação da Atenção (BPA). São Paulo SP: Vetor, 2013

SCHLINDWEIN-ZANINI R. Demência no idoso: aspectos neuropsicológicos. Revista Neurociencia, v. 18 n. 2 p. 220-26, 2010.

PAULA, J.; MALLOY-DINIZ, L. Teste de Aprendizagem Auditivo-Verbal de Rey (RAVLT). São Paulo: Vetor Editora Psico-Pedagógica Ltda, 2018.

SIÉROFF, É. La neuropsychologie: Approche cognitive des syndromes cliniques. 2 ed. Paris: Armand Colin, 2009.

SOUSA, L.; et. al. Neuropsicologia teoria e prática, 2. Ed. Porto Alegre: Artmed, 2014. p. 321.

SOUZA, V., et al. Perfil das habilidades cognitivas no envelhecimento normal. Revista CEFAC, v. 12, p. 2, 2010. 
SPREEN, O.; STRAUSS, E. A compendium of neuropsychological tests: Administration, norms and commentary: Oxford University Press. New York, 1998.

TEIXEIRA-JR, A.; CARDOSO, F. Neuropsiquiatria dos núcleos da base: uma revisão. Jornal brasileiro de psiquiatria, v. 53, n. 3, p. 153-158, 2004.

TRENTINI, C.; YATES, D.; HECK, V. Escala Wechsler Abreviada de InteligênciaWASI. Sao Paulo, SP: Casa do Psicologo, 2014.

VALLS-PEDRET, C.; MOLINUEVO, J.; RAMI, L. Diagnóstico precoz de la enfermedad de Alzheimer: fase prodrómica y preclínica. Revista de Neurologia, v. 51, n. 8, p. 471-480, 2010.

VALVERDE, A., et. al. A short neuropsychologic and cognitive evaluation of frontotemporal dementia. Clinical neurology and neurosurgery, v. 111, n. 3, p. 251255, 2009.

VAN DER MUSSELE, S., et. al. Depression in mild cognitive impairment is associated with progression to Alzheimer's disease: a longitudinal study. Journal of Alzheimer's Disease, v. 42, n. 4, p. 1239-1250, 2014.

VITTA A. Atividade física e bem-estar na velhice. In: A.L. Neri; S.A.Freire. (org.), E por falar em boa velhice. Campinas, SP: Papirus, p.25-38, 2000. (apud) FECHINE, Basílio Rommel Almeida; TROMPIERI, Nicolino. 0 processo de envelhecimento: as principais alterações que acontecem com o idoso com o passar dos anos. InterSciencePlace, v. 1, n. 20, p. 106-194, 2015.

YASSUDA, M., et al. Avaliação neuropsicológica de idosos: demências. Avaliação neuropsicológica. In: MALLOY-DINIZ Leandro F. et al. (org), Avaliação Neuropsicológica. Porto Alegre, RS: Artmed. 2010, p. 254-271.

ZHENG, D., et al. The overall impairment of core executive function components in patients with amnestic mild cognitive impairment: a cross-sectional study. BMC neurology, v. 12, n. 1, p. 138, 2012. 
ZIMERMAN, G. Velhice: aspectos biopsicossociais. Artmed Editora, 2009.

Enviado: Junho, 2020.

Aprovado: Setembro, 2020. 